$$
2316:-1
$$

UCRL-JC-125791

PREPRINT

\title{
Measured and Theoretical Characterization of the RF Properties of Stacked, High-Gradient Insulator Material
}

\author{
T. L. Houck
}

G. J. Caporaso, C. C. Shang, S. E. Sampayan, N. E. Molau

LLNL

M. L. Krogh

Allied Signal, Kansas City

This paper was prepared for submittal to the

1997 Particle Accelerator Conference

Vancouver, BC, Canada

May 12-16, 1997

\section{$5 / 9 / 97$}

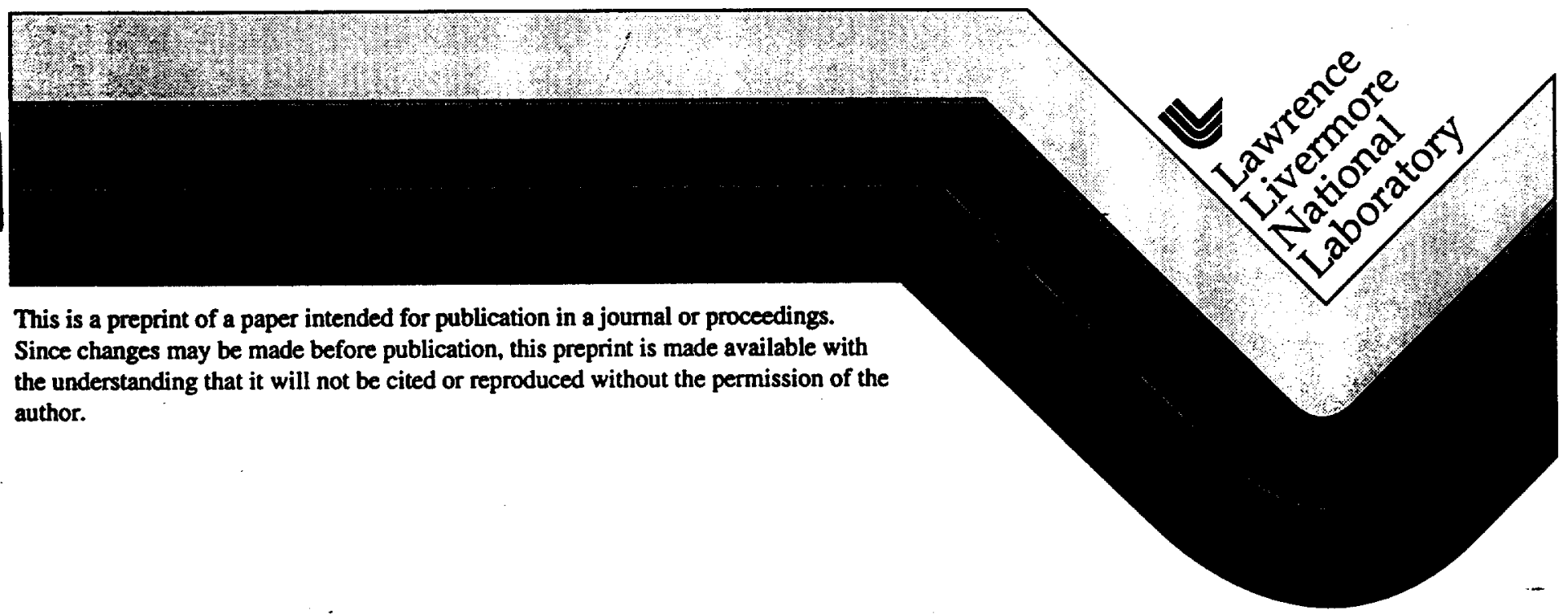




\section{DISCLAIMER}

This document was prepared as an account of work sponsored by an agency of the United States Government. Neither the United States Government nor the University of California nor any of their employees, makes any warranty, express or implied, or assumes any legal liability or responsibility for the accuracy, completeness, or usefulness of any information, apparatus, product, or process

disclosed, or represents that its use would not infringe privately owned rights. Reference herein to any specific commercial product, process, or service by trade name, trademark, manufacturer, or otherwise, does not necessarily constitute or imply its endorsement, recommendation, or favoring by the United States Government or the University of California. The views and opinions of authors expressed herein do not necessarily state or reflect those of the United States Government or the University of California, and shall not be used for advertising or product endorsement purposes. 


\title{
MEASURED AND THEORETICAL CHARACTERIZATION OF THE RF PROPERTIES OF STACKED, HIGH-GRADIENT INSULATOR MATERIAL
}

\author{
T.L. Houck, G.J. Caporaso, C.C. Shang, S.E. Sampayan, N.E. Molau \\ Lawrence Livermore National Laboratory, Livermore, CA 94551 \\ M.L. Krogh, Allied Signal Corporation, FM\&T, Kansas City, MO 64141
}

\section{Abstract}

Recent high-voltage breakdown experiments of periodic metallic-dielectric insulating structures have suggested several interesting high-gradient applications. One such area is the employment of high-gradient insulators in high-current, electron-beam, accelerating induction modules. For this application, the understanding of the of characteristics of the insulator plays an important role in estimating beam-cavity interactions. In this paper, we examine the rf properties of the insulator comparing simulation results with experiment. Different insulator designs are examined to determine their rf transmission properties in gap geometries.

\section{INTRODUCTION}

Insulators comprised of finely spaced, alternating layers of dielectric and metal have demonstrated greater (from 1.5 to 4 times) vacuum surface flashover capability than insulators made from a uniform dielectric $\left[{ }^{1},{ }^{2}\right]$. These high-gradient insulators (HGIs) have maintained similar capability in the presence of an electron beam $\left[^{3}\right]$. The high field stress level in the presence of a beam permits greatly simplify gap designs for induction accelerators. In typical induction accelerator designs $\left[{ }^{4}\right]$, the gap is shaped to accommodate an extended insulator hidden from the beam line-of-sight view The gap shape must then be carefully designed to minimize field stress. A simplified gap design using the superior performance of the HGI technology has been suggested for the induction modules for a proposed TBNLC rf power source $\left[{ }^{5}\right]$.

Collective beam instability associated with the gap's transverse beam impedance is of equal importance to surface flashover. Transverse impedance scales linearly with the gap width and inversely with the square of the beam line aperture $\left[{ }^{6}\right]$. Increasing the aperture to lower impedance is effective, but is not energy or cost efficient. HGI technology, by minimizing gap width and less complicated gap designs, can reduce the induction core volume to achieve higher efficiency.

A concern with the use of the HGI in gap designs is the possible introduction of high-impedance resonances from the more complicated geometry of the insulator. Transverse impedance measurements were performed with HGI insulators positioned at the mouth of simplified cavity gaps. These measurements were then compared against solid insulators.

\section{EXPERIMENTAL LAYOUT}

The impedance measurements were performed by a standard technique $\left.{ }^{7}\right]$ where the beam pipe is transformed into a coaxial TEM transmission line by inserting two center conductors and measuring transmission through the two-wire transmission line. The center conductors are driven through a $180^{\circ}$ hybrid to excite transverse currents in the test cavity. Transmission through the beam line with the a test cavity inserted is compared to that of a smooth pipe of equal longitudinal length as the test cavity. Measurements of the Sparameters were made with an HP8510C Network Analyzer. The through-short-delay (TSD) calibration technique was used to remove the effect of the matching sections between the analyzer and beam pipe transmission line. The real part of the transverse impedance can be calculated from the measured S-parameters using [ []

$$
\begin{aligned}
& Z_{\perp}=\frac{2 Z_{c}}{\omega \Delta^{2}}\left(\frac{\cos \theta}{S}-1\right) \text {, where } \\
& S=\left|\frac{S_{21}}{S_{21}^{\text {ref }}}\right| \text {, and } \theta=\arg \left[\frac{S_{21}}{S_{2 !}^{\text {nf }}}\right] \text {, and }
\end{aligned}
$$

$\Delta=10.8 \mathrm{~cm}$ is the wire separation. The superscript "ref" indicates the value measured for a smooth pipe, or reference, configuration. The characteristic impedance of the two-wire transmission line, $\mathrm{Z}_{\mathrm{c}}$, had a value of $359 \Omega$ for the configuration used

The test cavity represented a simplified induction module. The cavity geometry was a pillbox separated into two regions by a $3 \mathrm{~mm}$ thick ring of inside diameter of $23.4 \mathrm{~cm}$, concentric with the cavity axis, and extending $2.25 \mathrm{~cm}$ across the cavity. A $6 \mathrm{~mm}$ thick, $2.54 \mathrm{~cm}$ wide ring of ferrite (TDK PE11B) was positioned between the OD of the ring and the outer wall of the cavity. The ferrite was effective in reducing the $Q$ of the trapped dipole modes to less than 3, a typical value for induction accelerator gap designs. A schematic of the test cavity is shown in Fig. 1.

The insulators were comprised of thin disks $(0.5 \mathrm{~mm})$ of polycarbonate or stainless steel, with inner diameter of $14.6 \mathrm{~cm}$ and outer diameter of $19.7 \mathrm{~cm}$ or $22.2 \mathrm{~cm}$ depending on the configuration. The distribution of disks was varied to produce different ratios of insulator to conductor and different number of alternating layers, or periods, for a specific ratio. A photograph of the pillbox test cavity with a 4:1 ratio stacked insulator is shown in Fig 2. One wall of the cavity was removed for the picture. 


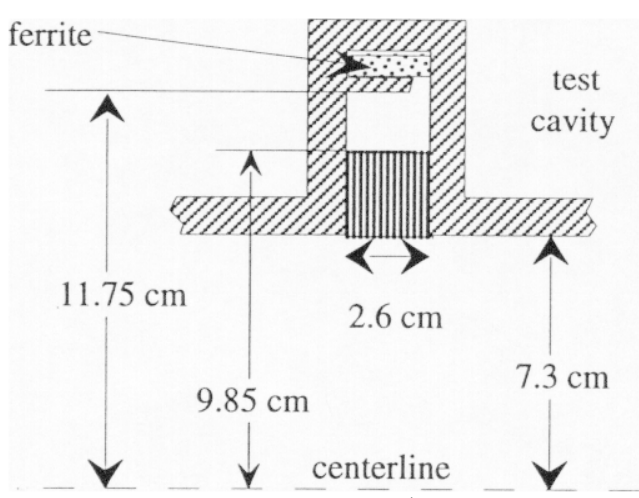

Figure 1. Schematic of the pillbox test cavity.

\section{EXPERIMENTAL RESULTS}

Impedance measurements of the test cavity with the $19.7 \mathrm{~cm} \mathrm{OD} \mathrm{insulator} \mathrm{and} \mathrm{different} \mathrm{ratios} \mathrm{of} \mathrm{dielectric} \mathrm{to}$ conducting material is shown in Fig. 2. The smaller peaks flanking the primary resonance at $1.35 \mathrm{GHz}$ are related to the geometry of the transmission line and are not specific to the test cavity. Addition of microwave absorbers at the ends of transmission line pipe reduced these impedances while not affecting the primary or $350 \mathrm{MHz}$ resonances. The TE/TM cutoff frequencies for traveling wave modes in the transmission pipe are $1.2 / 1.57 \mathrm{GHz}$ respectively. The $350 \mathrm{MHz}$ resonance is not affected by the structure of the insulator. Removal of the intermediate ring in the pill box also did not significantly change the impedance of this resonance, although the $1.35 \mathrm{GHz}$ resonance was effectively removed. The number of layers, or periods, of polycarbonate were 9 for the $2: 1,7$ for the $1: 1$, and .9 for the $1: 2$ ratios respectively. Data in Fig. 3 can be directly compared with computer simulations shown in Fig. 6.

Results for the $22.2 \mathrm{~cm}$ OD insulator are shown in Fig. 4. The impedance measurements are similar to the smaller OD insulator so an expanded view of the impedance spectrum around the primary resonance is given. The number of periods of insulator were 11 for the $4: 1$ ratio, 7 for the 3:1 ratio, 9 for the $2: 1$ ratio, and 7 for the $1: 1$ ratio. The affect of increasing the number of periods while maintaining the same ratio of dielectric to stainless steel is shown in Fig. 5. The impedance converged rapidly with increasing number of periods.

\section{THEORETICAL CALCULATIONS}

Impedance calculations were performed using a 2.5-D, full-wave, time-dependent, wakefield code with a dispersive model for the PE11b ferrite [9]. Two series of calculations were performed for the geometry of Fig. 1.

Fig. 6 displays the dipole impedance spectrum calculated by AMOS for a nominal solid insulator, and simulated stacked insulators with 2:1, 1:1, and 1:2 dielectric to metal thickness ratios. These results not only mimic the physics observed in the experiment, but also are in excellent quantity agreement with the experiment.

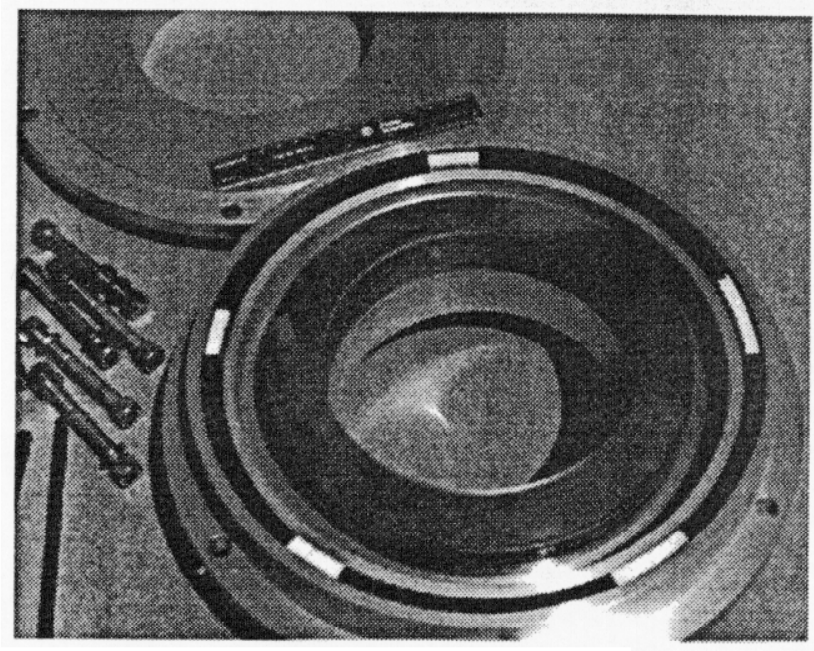

Figure 2. Photograph of test cavity with insulator. One wall of the cavity has been removed so that the insulator, intermediate ring, and damping ferrite are visible.

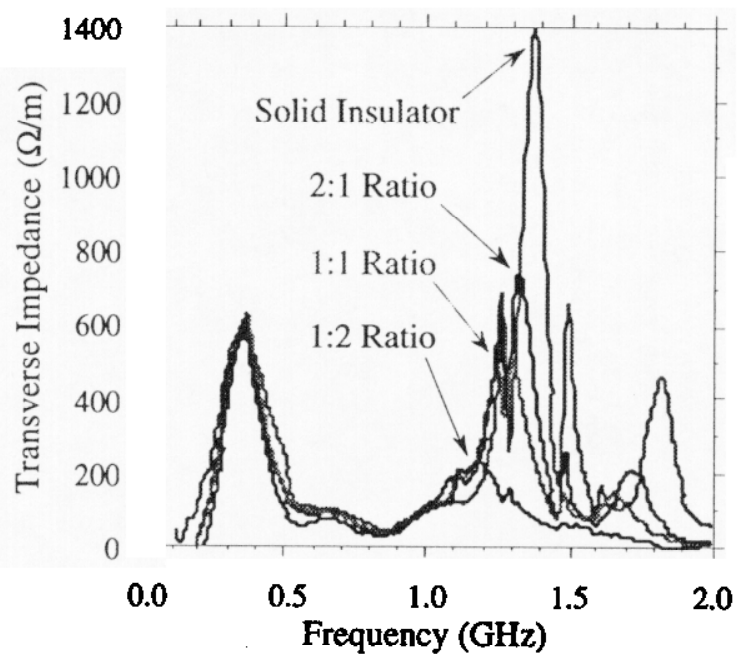

Figure 3. Impedance spectrum of the cavity with $19.7 \mathrm{~cm}$ $\mathrm{OD}$ insulator for different dielectric and conductor ratios.

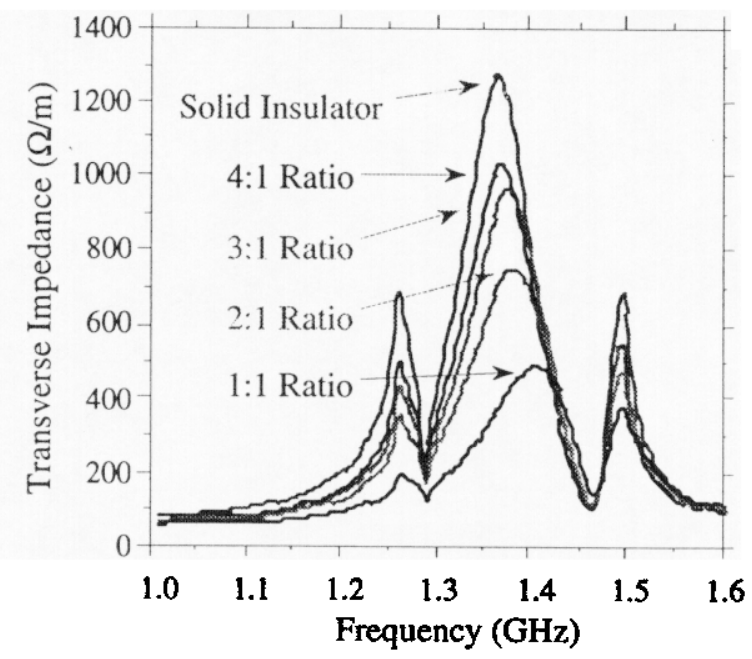

Figure 4. Effect on the primary resonance due to varying the ratio of dielectric to conducting material. 


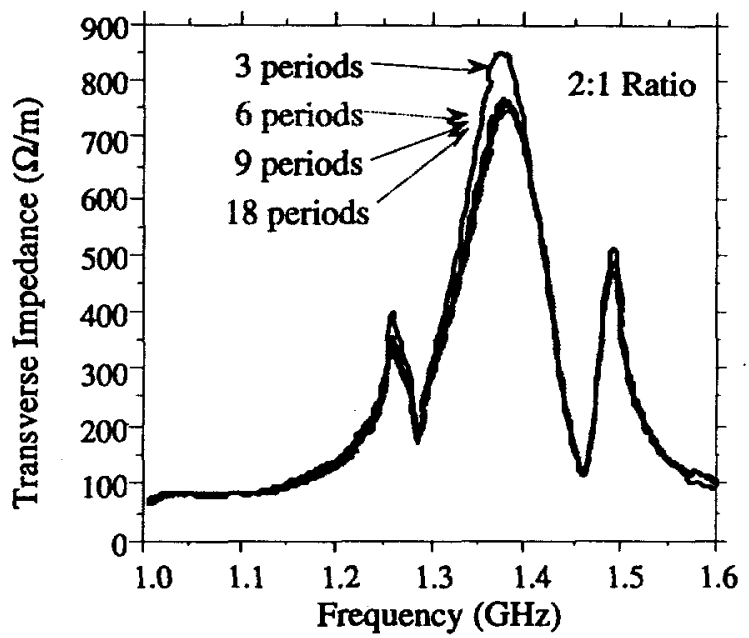

Figure 5. Effect on the primary resonance due to increasing the number of periods while maintaining the ratio of dielectric to conducting material.

Figure 7 illustrates the rapid convergence to a minimal impedance value for a fixed dielectric to conductor ratio as the number of layers,or periods, of dielectric is increased. Both experimental and theoretical predictions are consistent with the observation that the effect we see is a mode dependent and not a frequency dependent phenomenon.

\section{CONCLUSIONS}

Observations that can be made from the experimental measurements and simulations include:

(1) Geometry of the stacked insulator does not result in new resonances of significant impedance.

(2) The ratio of dielectric to conducting material is more important than the number of layers in the insulator in regards to the effect on impedance.

(3) For a specific ratio, the impedance converges rapidly to a final spectrum with increasing number of periods.

(4) AMOS accurately calculates the impedance spectrum associated with stacked insulators.

(5) To first order, the reduction of impedance can be related to the reduction in effective gap (dielectric) width.

(6) A possible second effect of the stacked insulator is a shifting of resonance field patterns leading to more effective damping by absorbers.

\section{ACKNOWLEDGMENTS}

We thank D. Prono (LANL), M. Burns (LANL), and R.E. Clough (LLNL) - for their encouragement and support. We also thank C. Holmes for fabricating the test cavity and J. Dunlap for assistance with the impedance measurements. The work was performed under the auspices of the U.S. Department of Energy by Lawrence Livermore National Laboratory under contract W-7405ENG-48 and by Allied Signal Corp. under contract DEAC04-76-DP00613.

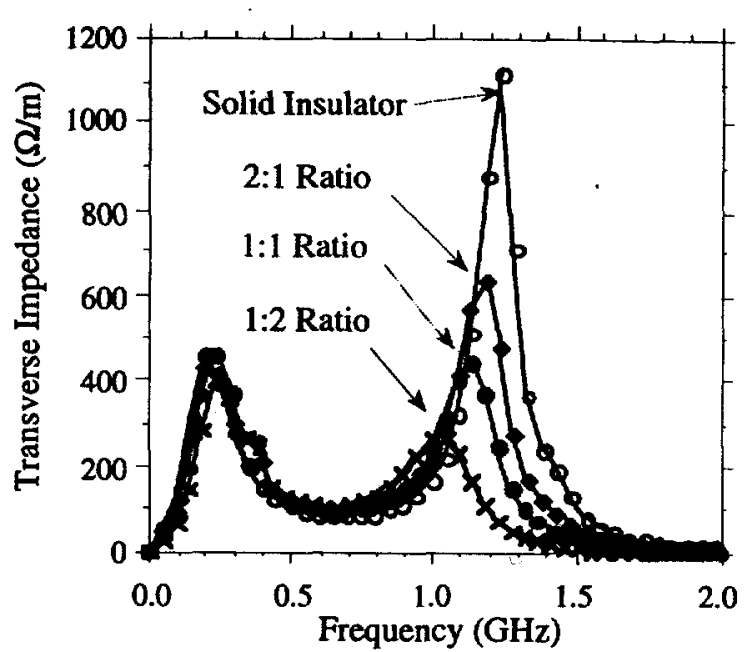

Figure 6. Impedance spectrum from AMOS calculations for actual experimental measurements. Refer to Fig. 3.

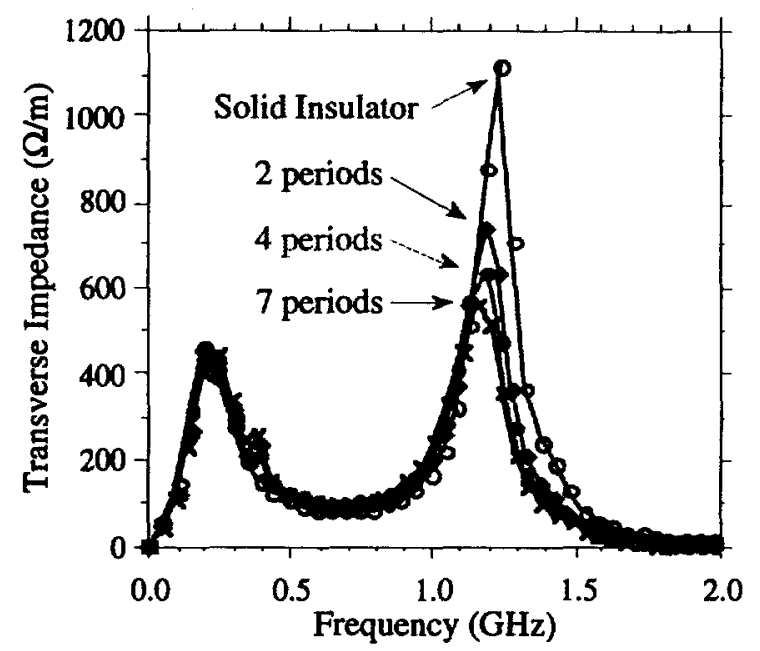

Figure 7. Simulation results illustrating the rapid convergence of the impedance spectrum with increased number of layers, or periods, of insulating material.

\section{REFERENCES}

[1] Juan M. Elizondo and A.E. Rodriguez, "Novel High Voltage Vacuum Surface Flashover Insulator Technology," Proceedings of the XVth International Symposium on Discharges and Electrical Insulation in Vacuum, Vde-Verlag, Berlin, Germany, pp. 198-202 (1992).

[1] S. Sampayan, et al., "High-Performance Insulator Structures for Accelerator Applications", these proceedings.

$\left[{ }^{3}\right]$ S. Sampayan, et al., Proceedings 1995 Particle Accelerator Conference, (IEEE), New York, N.Y., pp. 1269-1271.

['] M. Burns, et al., Procedings 1991 Particle Accelerator Conference, (IEEE), New York, N.Y., pp. 2958-2960.

[5] J-S Kim, et al., "Stack Insulator Induction Gaps", these proceedings [6] R.J. Briggs, et al., Part. Acc., 18, pp.41-62, 1985.

[7] L.S. Walling, et al., Nucl. Inst. Meth., A281 (1989) 433.

${ }^{8}$ ] L.S. Walling et al., Proceedings of the 1990 LINAC, p. 704.

['] J.F. Deford and G. Kamin, Proceedings of the 1990 LINAC, p. 384. 


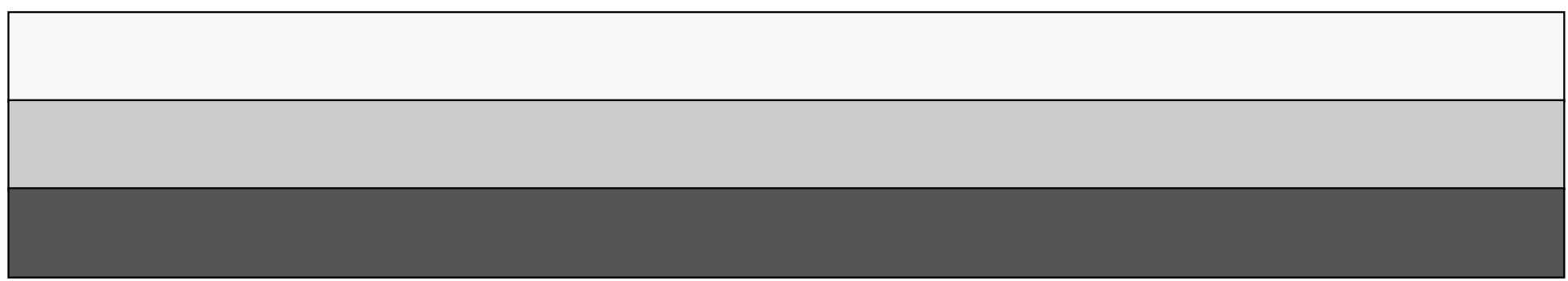

